\title{
Efficient Coverage Algorithm Base on Node Scheduling Strategy in Wire- less Sensor Network
}

\author{
Xiaoguang Li and Xucan Bai
}

Department of Electrical Engineering and Automation, Luoyang Institute of Science and Technology, Luoyang, Henan, 471023, China

\begin{abstract}
Maximize coverage and prolong the network lifetime for wireless sensor networks has become one of the key topics of research. For this, put forward a kind of node scheduling strategy based on covering algorithm. The algorithm by Poisson distribution model structure node density formula, according to the node density formula in the monitoring region deploying to effective coverage; On the other hand, through the node state scheduling mechanism updates as well as to the neighbor node, can be made of residual energy of nodes and monitoring the area sensor the node energy consumption balance, so as to prolong the network lifetime goal. The simulation results show that, the algorithm cans not only useless nodes completely too effective coverage, improve the coverage while optimizing the cyber source configuration, prolong the network lifetime.
\end{abstract}

Keywords: Wireless sensor network, Network lifetime, Coverage rate, Nodes.

\section{INTRODUCTION}

Wireless sensor network with other network there are certain differences in the architecture. Firstly, in terms of the wireless sensor network itself, and it is through the dense nodes of monitoring area control, sensor nodes are smaller and energy [1,2], LTD; In some of the more severe and dangerous environment, the energy of the sensor nodes and can't effectively supplement; Second, in the high-density deployment of monitoring area, when in the working state of multiple sensor nodes to a particular target monitoring, location, coverage and tracking, will lead to multiple data collected by the sensor node or calculated information to a neighbor node and generated when the base station sends a large number of redundant data, it will inevitably cause the network congestion, leading to a large number of useless sensor node energy consumption, will eventually lead to the total energy of the wireless sensor network (WSN) too quickly consumption, reduces the amount of effective network life cycle. Thirdly, in the process of practical application, not all of the target node should be completely covered. That a node is the focus on target node, which begs to effectively cover completely cover or $K$ degree, that is, the destination node in a sensor node $K$ perceived scope. When the destination node is not concern, let their perception in a sensor node scope. In the process of cover, still need to consider in a certain conditions, some sensor nodes in a dormant state, in order to effectively guarantee the network energy, prolong the network life cycle.

\section{RELATED WORKS}

In recent years, the coverage problem in wireless sensor network has been concerned by scholars in the world. Scholars have carried out some very fruitful research points. Literature gives the monitoring areas of all sensor nodes are divided into a number of disjoint subsets, each subset in a monitoring area conduct independent monitoring, through the clock timing function for each subset of alternate methods for monitoring area of the target node to effectively cover [3]; the authors using a self calibration method for a particular node redundant nodes into dormancy, to avoid the blind spots are round monitoring, after a specified period of time, the active node updates the local Voronoi to complete the map of monitoring regional coverage [4]; On wireless sensor networks heterogeneous nodes network topology, all nodes in monitoring area cluster processing, make high energy sensor nodes form a cluster head node, on the formation of cluster regional monitoring and coverage [5]; the paper is the use of wireless sensor network having distributed characteristics will detect and control integration, to the fused sensor node subset optimization, to save energy, prolong network cycle [6]. Literature gives an energy saving cover, the idea is to use each sensor node of the perceived distance equilibrium conditions to achieve energy-saving coverage, this method is not random mobile sensor nodes [7]. The using density control PEAS algorithm to schedule the target area sleep node and in different regions of the sensor node energy consumption itself as far as possible, to prolong the network lifetime [8]. All of the above algorithm is to a large extent can be completed the coverage and connectivity, but the solving process is too complex; in addition, with the increase of the number of sensor nodes and coverage area change, also can make the algorithm 


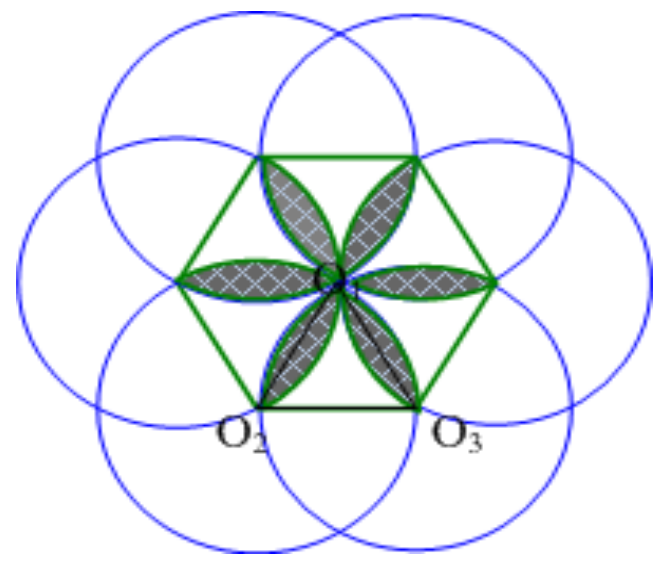

Fig. (1). Any intersection of two circles.

complexity is increased, thereby reducing the computational efficiency. The above six algorithms in different degree to satisfy a condition coverage requirements, but also can guarantee the connectivity of the network, but its disadvantage is node distribution unevenness caused the complexity of the algorithm is too high, speed too slow, and a plurality of sensor node position changes will occur more frequently perceived area multiple cover the possibility [9, 10].

\section{MODEL ANALYSIS}

\subsection{Network Model and Hypothesis}

The following hypotheses are advanced on the network model:

Hypothesis 1: The monitored area is much larger than the sensor node sensing area, not considering the boundary factors on the monitoring of regional influence.

Hypothesis 2: Sensor node sensing radius and radius of communication will appear a disk shape and the communication radius greater than or equal to 2 times the radius of perception.

Hypothesis 3: Each sensor node can be through their own information to their location information.

Hypothesis 4: The initial state, and each sensor of node energy is the same, all sensor nodes have the same processing capacity, and equal status.

\subsection{Basic Definition}

Definition 1: The distance between any two nodes $d(i, j)$ are called nodes $i$ and $j$ Euclidean distance, when $d(i, j)<2 \mathrm{R}$ referred to the neighbor node, node $i$ and $j$.

Definition 2: In the monitoring of the target area, when a target node is $K$ sensor node coverage, called $K$ heavy cover.

Definition 3: In the monitoring of the target area, all sensor nodes coverage Union and all sensor nodes range and than, called network covering efficiency:
$E A=\underset{N=1,2 \ldots N}{\mathrm{U}} S_{i} / \sum_{N=1,2 \ldots N} S_{i}$

Definition 4: Covering the region of coverage for:

$p\left(s_{i}, s_{j}\right)= \begin{cases}0 & \text { if } R_{s} \leq d\left(s_{i}, s_{j}\right) \\ e^{-\varepsilon d} & \text { if }\left(R_{s}-R_{e}\right)<d\left(s_{i}, s_{j}\right)<R_{s} \\ 1 & \text { if } d\left(s_{i}, s_{j}\right) \leq\left(R_{s}-R_{e}\right)\end{cases}$

Among them: $\varepsilon$ is sensor node physical parameters; $R_{e}$ said sensor node monitoring dynamic parameters in the said sensor nodes; $d\left(s_{i}, s_{j}\right)$ Euclidean distance; when $d\left(s_{i}, s_{j}\right)<\left(R_{\mathrm{s}^{-}}\right.$ $R_{\mathrm{e}}$ ), this time node $S_{\mathrm{i}}$ is detected, it is not detected [11-13].

Theorem 1: When and only when the three equal circles intersect at one point, and form an equilateral triangle length of a side is $\sqrt{3}$, covering the efficiency of $E A$ maximum, That is: $\mathrm{EA} \leq 82.73 \%$

Proof: As shown in Fig. (1):

Firstly, Fig. (1) were analyzed, as are three. Two intersect, and the intersection region are equal, so $\mathrm{VO}_{1} \mathrm{O}_{2} \mathrm{O}_{3}$ is an equilateral triangle, with side length $O_{1} O_{2}$ is $r$, an equilateral triangle $\mathrm{VO}_{1} \mathrm{O}_{2} \mathrm{O}_{3}$ three interior angles are respectively $\pi / 3, \angle O_{2} O_{1} O_{3}=\pi / 3, S_{\mathrm{VO}_{1} O_{2} O_{3}}=\frac{1}{2} r^{2} \sin \pi / 3$, since three, round two intersection, and completely covered on the plane the Euclidean distance, $d_{\mathrm{i}}<2 r$, let the equilateral triangle $\mathrm{VO}_{1} \mathrm{O}_{2} \mathrm{O}_{3}$ the maximum length to keep the $\mathrm{S}_{\mathrm{VO}_{1} \mathrm{O}_{2} \mathrm{O}_{3}}$ area is the largest, the three circle intersect at a point $B$, as shown in Fig. (1) as shown, connect to the $O_{3} B$ and extended to two points to $A$, connecting the $\mathrm{O}_{2} A$, set three the radius of the circle of $1,{S V_{A B O}}_{2}=\sqrt{3} / 4, S_{\mathrm{ABO}_{2}}=\pi / 6$, according to the formula (1) we get $E A=S_{\mathrm{V}_{A B O} O_{2}} / S_{A B O_{2}}$, $E A=3 \sqrt{3} / 2 \pi=82.73 \%$ namely in the completely covered cases the maximum coverage of the efficiency value is $82.73 \%$. 


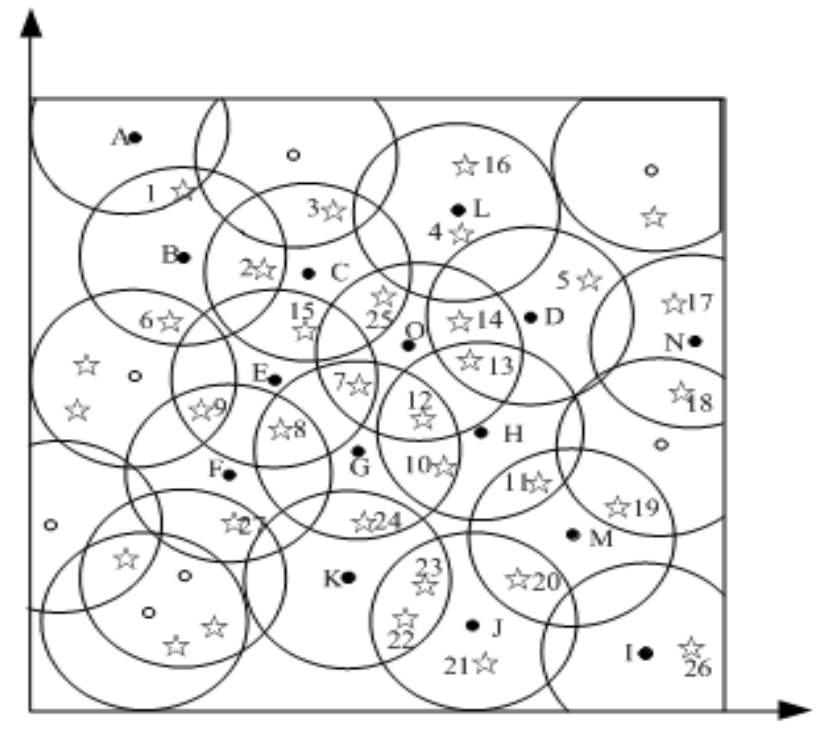

Fig. (2). Network coverage models.

Theorem 2: Sensor node monitoring area $A$, the monitoring of regional node density $\lambda$, a monitoring area $A$ node number $X$ subject to node $K$ probability density:

$P(X=k)=e^{-\lambda A} \cdot(\lambda A)^{k} / k !$

Proof: The monitoring area is $S$, in the monitoring region of arbitrary nodes subordinated to the $K$ node distribution probability of $P=A / S$, when the number of nodes of $n$ probability obeys two type distribution is:

$P(X=k)=C_{n}^{k} p^{k}(1 \quad p)^{n-k}$

According to the node density formula $\lambda=n / S$ into arbitrary node distribution probability of $P$ :

$P=A \lambda / n$

Equation (5) into the formula (4):

$$
\begin{aligned}
P(X=k) & =C_{n}^{k}(A \lambda / n)^{k}(1 \quad A \lambda / n)^{n-k} \\
& =\frac{n !(A \lambda)^{k}(1-A \lambda / n)^{n}}{(n-k) ! k !(n-A \lambda)^{k}}
\end{aligned}
$$

When $n \rightarrow \infty$ and its limit available:

$$
\begin{aligned}
P(X=k) & =\lim _{n \rightarrow \infty}\left(\frac{n !(A \lambda)^{k}(1-A \lambda / n)^{n}}{(n-k) ! k !(n-A \lambda)^{k}}\right) \\
& =e^{-\lambda A}(A \lambda)^{k} / k !
\end{aligned}
$$

That is: $P(X=k)=e^{-\lambda A} \cdot(\lambda A)^{k} / k !$

\section{COVERAGE CONTROL AND SCHEDULING OF NODES}

When the focus of the target node is considered to be the key target, it will be covered with multiple sensor nodes, in order to achieve the purpose of the cover and positioned accurately. Each node in the process of cover, for a certain probability to choose their own work nodes, there're a total of five kinds of state covering process can convert each other, constitute the node scheduling strategy. As shown in Fig. (2).

Diagram shows the sensor nodes in the process of monitoring area covering the target node data information obtained through the perception scope, by calculation and forwarded to neighbor nodes or anchor node; At the same time using multiple sensor node to destination node formed by multiple coverage to determine the focus of the target node, the coverage is greater than or equal to $2[14,15]$.

\subsection{Dynamic Form}

When the target into a cluster head monitoring area, to the neighbor cluster head node sends a packet containing the target information, all the monitoring to the target cluster are dynamically in the target around to form a group, cluster member nodes only with the cluster node communication, the cluster head and between cluster heads can be mutually communication. Involved in tracking the cluster number depending on the size of the radius of the grid. For example, if the access grid side length equal to the radius of communication node, then a maximum of only four cluster capable of simultaneously monitoring to the target. When at the same time two or more than two cluster head and monitoring to the target, we select these clusters in a cluster head node as a leader node, cluster head first to the neighbor hair to send their and monitoring the distance between the target data information, if the cluster head received a distance closer to the target hair to information, give up campaign to become leader node. Selection criteria for : first, choose from the closest cluster head node; second, if there are two or more than two cluster head node and the target 


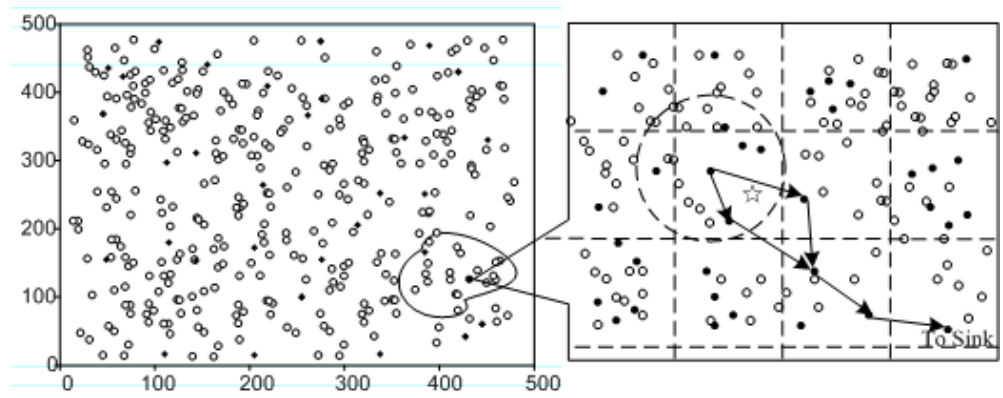

Fig. (3). The target node coverage area diagram.

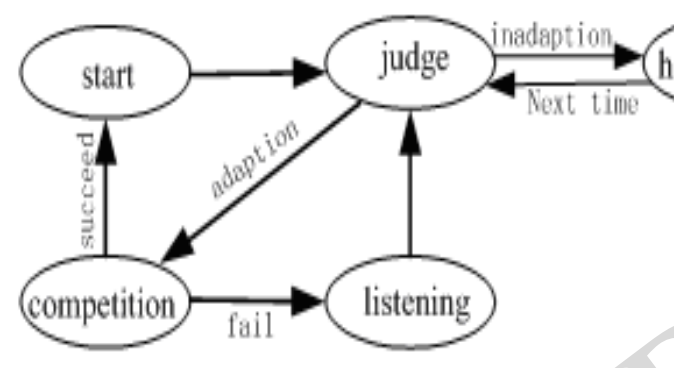

Fig. (4). Node scheduling state diagram.

and the distance between the same, residual energy larger the lead node. All the monitoring to the target cluster head node will be sent to a leader node data first, and then by the leading node calculation and data fusion are transmitted to a data center node. As shown in Fig. (3):

When the mobile target leading away from the node, because of the need to transmit data over long distances to the leader node, or a new cluster head node monitoring to the target, then a leader node is no longer applicable acts as a leader node, fast the election of a new leader node is very necessary. Here we shall, when there is a new cluster head node joins the mobile target tracking, under the leadership of node selection rules, in all involved in tracking the cluster head node selects a distance to a target the nearest cluster head node as its new leader node, data reported by the new leader node is sent to a data center [16].

\subsection{Node Scheduing Strategy}

The sensor node is a round number as the cycle to work. During an initialization phase, the sensor node closed its induction module, update their information and the neighbor node. In the scheduling process to go through five states, respectively, the start state, judge state of competition state, hibernation, the listening state, a five state conversion constitute the sensor node scheduling strategy. First of all, to judge if the node meet the dormancy condition, such as meet into hibernation, or into the competitive status, when entering into competition, start a timer; secondly, when the node competitive success, node to the start state, competition failure node into the listening state; again, in a sense node on success to receive the neighbor node to broadcast news
On-duty Message, update its neighbor nodes' information, thus entering the judgment condition; fourth, in the starting state of the node to its neighbor node sends a On-duty Message, which contains the start node only ID identification and location information, and carries on the effective coverage of the work; fifth, in order to save the energy of the node, for accurate monitoring region to effectively cover, the sensor node will turn off unnecessary device to prolong the network life cycle [17-19]. In practical application process, the sensor node according to the neighbor node's information to dispatch their information, until sure sensor node itself as the start state or resting state so far, as shown in Fig. (4).

\section{SYSTEM ASSESSMENT}

Wireless sensor network node energy consumption most are from to in data processing and communication, contains the main data calculation, data processing and data transmission on three aspects, including data transmission by the consumption of energy is higher, the wireless communication consumption model was studied, its model:

$$
\begin{aligned}
& E_{T r}(k, d)=E_{T-\text { elec }} k+E_{\text {amp }}(k, d) \\
& =\left\{\begin{array}{cc}
E_{T-\text { elec }} k+\varepsilon_{f s} d^{2} k & d<d_{0} \\
E_{T-\text { elec }} k+\varepsilon_{\text {amp }} d^{4} k & d \geq d_{0}
\end{array}\right.
\end{aligned}
$$

$E_{R x}(k)=E_{R-\text { elec }} k$

On the type, $\mathrm{E}_{\mathrm{T} \text {-elec }}$ and $\mathrm{E}_{\mathrm{R} \text {-elec }}$ wireless sending and receiving module of energy consumption; $\varepsilon_{\mathrm{fs}}$ and $\varepsilon_{\mathrm{amp}}$ said free space model and the multiple attenuation model amp consumption parameters; $\mathrm{d}_{0}$ is constant. 


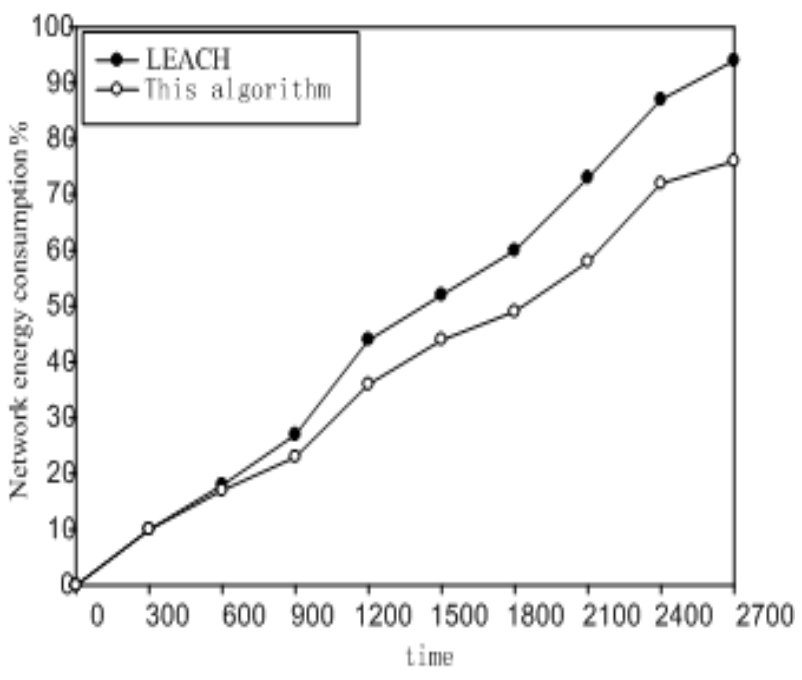

Fig. (5). Network energy diagram.

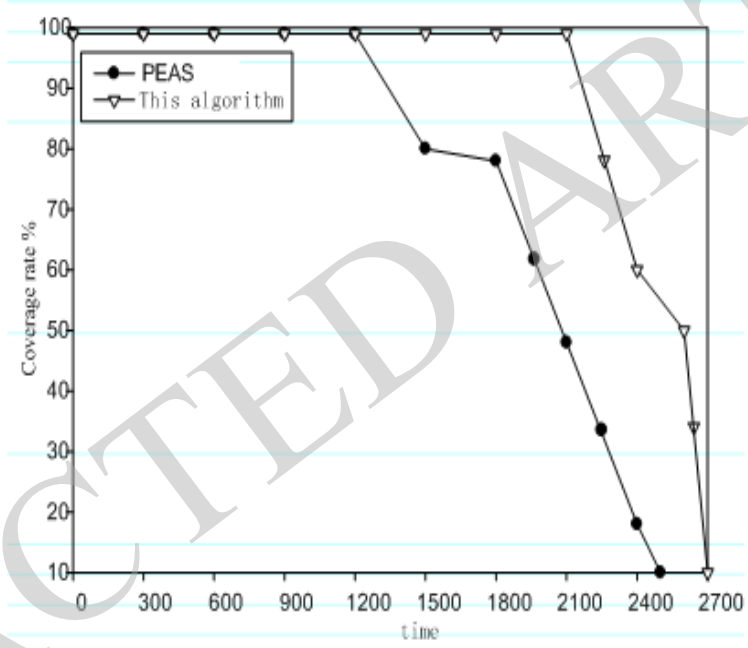

Fig. (6). Different rounds of the network coverage.

In order to verify the effectiveness of the algorithm and stability, the experiment from the two aspects of the comparison, the simulation platform is MATLAB7, the simulation region is set to $500 \mathrm{~m} \times 500 \mathrm{~m}$, sensor nodes is 300 , sensor node sensing radius is $5 \mathrm{~m}$, the initial each node's energy is the same $2 \mathrm{~J}$, network bandwidth is $1 \mathrm{Mbps}$, packet size is $100 \mathrm{bits}$, the sampling period is $10 \mathrm{~s}$.

The first case: the algorithm and LEACH protocol comparison experiment, validation in the same round number, the network energy consumption contrast conditions, as shown in Fig. (5).

From the Fig. (4) can be seen, in the initial moments, two algorithms of the network energy consumption approximately equal; but as time goes on, the algorithm of the network energy consumption less than LEACH protocol of energy consumption, and this algorithm to the subsequent time tends to be stable, in the entire network cover cycle, this algorithm can effectively save the sensor node energy, prolong the network life cycle.

In second cases, using this algorithm with PEAS algorithm in coverage on the comparison experiment, the coverage rate of $99 \%$ that is completely covered, the experimental data are in 100 simulation data by extracting the average value, as shown in Fig. (6).

From seeing the graph 5, as time progresses, two algorithms of coverage has declined. The initial phase, two algorithms of coverage, but in $\mathrm{t}=1500$, PEAS algorithm coverage declined more obvious, the algorithm still maintain higher coverage. In the same node under the effect of this algorithm, coverage was significantly higher than that of PEAS algorithm, to verify the effectiveness of this algorithm. 


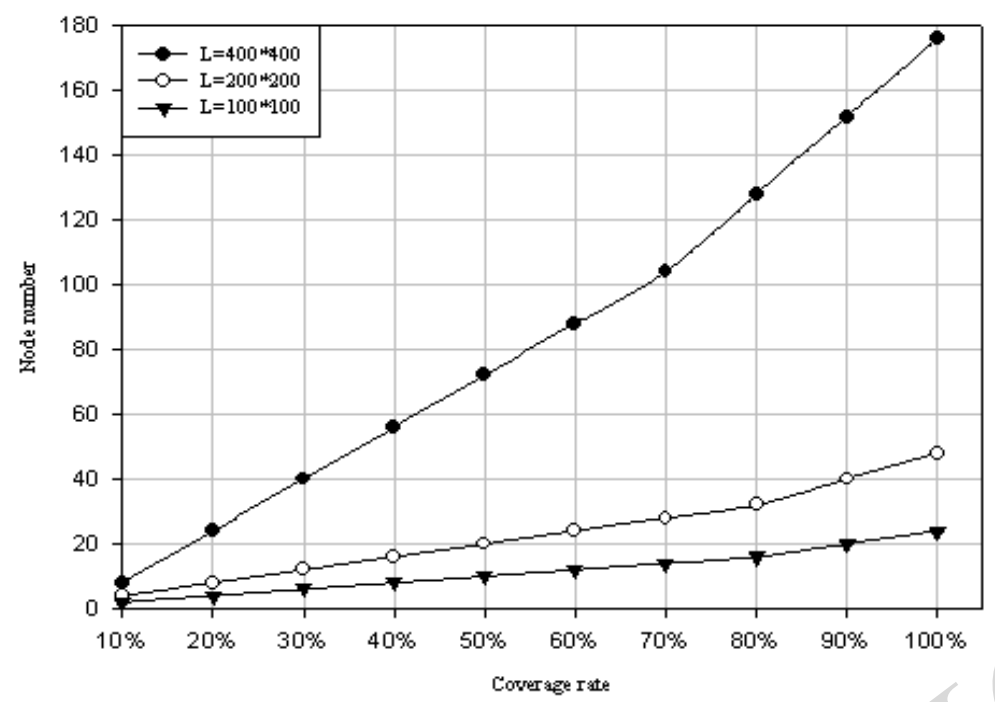

Fig. (7). Coverage rate for different coverage area.

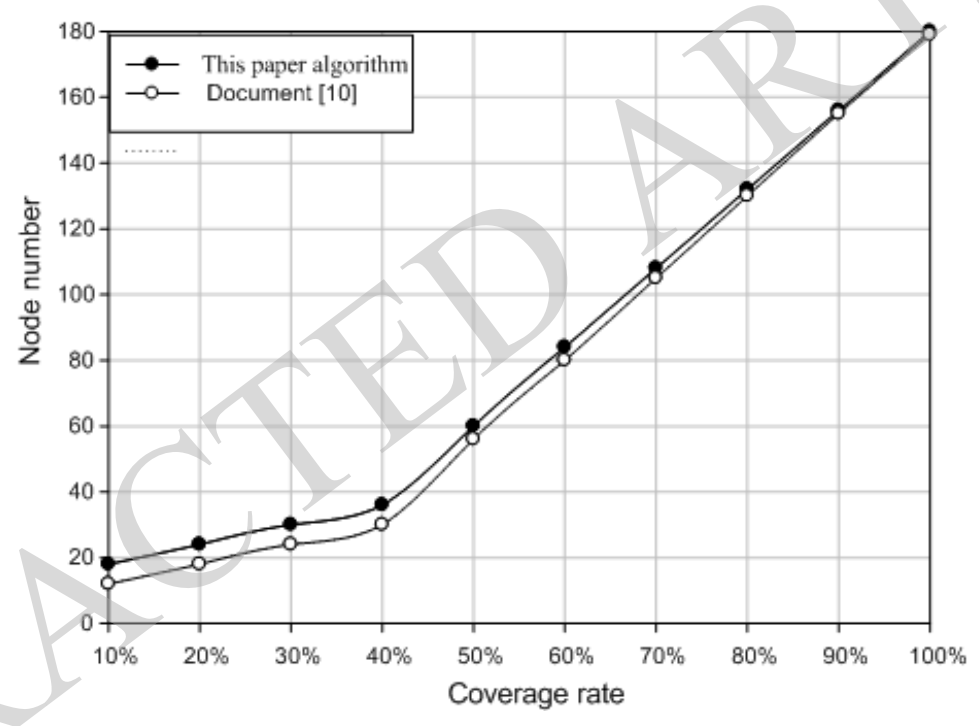

Fig. (8). Coverage comparison.

In order to achieve the scale of network coverage, and thus better evaluate the performance of the model in different sizes, which mainly reflect the minimum number of nodes needs to by deploy in different network coverage, each simulation experiment executed 50 times at average. Curve of node coverage changes is shown in Fig. (7).

Fig. (7) shows the graph of the number of sensor nodes needed to deploy to achieve different node coverage under different network dimensions. The figure shows that, with the expansion of the network, to meet the demand for network coverage, the number of nodes required to be deployed will increase, and the higher the coverage of the network, the number of nodes need to be deployed increases can be obtained from Figure more fast, so that the concern target node can achieve complete coverage.
Experiment: Fig. (8) shows a diagram of the number of sensor nodes need to be deployed for the same network size $400 * 400 \mathrm{~m}^{2}$ under different node coverage requirement, and compare with the experiments of literature SCCP algorithm, to meet certain demand for network coverage, the number of nodes deployed will be gradually increased as time progresses, and the network coverage will also increase, so that completely coverage is achieved for the same coverage area and different nodes coverage for target area, as shown in Fig. (8).

\section{CONCLUSION}

This paper studies the coverage problem in wireless sensor network, using the node to node state transition mechanism for effective control, giving the Poisson 
distribution model and reasoning the joint probability density, and verifying the efficiency of coverage, quantifying the node density distribution, optimizing the network resources. Using relationship between the sensor node and the target node, it gives the coverage model of monitoring region network, identifies the sensor node and the target node affiliation, as well as the effects that $K$ heavy coverage puts on the network monitoring region. Using the node state transition mechanism, it constructs the node wheels work system model. Finally through the simulation experiment on the LEACH protocol and PEAS algorithm comparison experiment, in the network energy consumption and network coverage rate of this algorithm, this paper is higher than above two algorithms, achieving the goal of prolonging the network lifetime and improving the quality of network service.

\section{CONFLICT OF INTEREST}

The authors confirm that this article content has no conflicts of interest.

\section{ACKNOWLEDGMENTS}

This work is supported by the Science of Technology Research of Foundation Project of Henan Province Education Department under Grant Nos. 2014B520099, and Natural Science and Technology Research of Foundation Project of Henan Province Department of Science under Grant Nos. 142102210471 .

\section{REFERENCES}

[1] S. Slijepcevic, and M. Potkonjak, "Power efficient organization of wireless sensor networks," IEEE Conference on Communication vol. 6, no. 2, pp. 472-476, 2001.

[2] B. Carbunar, A. Grama, and J. Vitek, "Redundancy and coverage detection in sensor network," ACM Transactions on Sensor Network, vol. 2, no. 1, pp. 94-128, 2006.

[3] V. Mhatre, and C. Rosenberg, "A minimum cost heterogneous sensor network with a lifetime constraint," IEEE Transaction on Mobile Computing, vol. 3, no. 3, pp. 27-31, 2004.

[4] N. Ruixin, K. Pramod, and Q. Varshney, "Distributed detection in a large wireless sensor network," Information Fusion, vol. 7, no. 4, pp. 380-384, 2006.
[5] Y. Jin, and J. Jo, "An energy efficient coverage and connectivity preserving routing algorithm undeer border effects in wireless senson networks," Computer Communications, vol. 33, no. 10, pp. 2398-2407, 2008.

[6] W. Ke, and B. Liu, "Efficient algorithm for constructing minimum size wireless sensor networks to fully cover critical square grids," IEEE Transactions on Wireless Communications, vol. 10, no. 4, pp. 1154-1164, 2011.

[7] D. Dong, and X. Liao, "Distributed coverage in wireless Ad Hoc and sensor networks by topogical graph approaches," IEEE Transactons on Computers, vol. 61, no. 10, pp. 1417-1428, 2011.

[8] H. Ammari, and S. Das, "Centralized and Clustered K-coverage protocols for wireless sensor network," IEEE Transactions on Computers, vol. 6, no. 1, pp. 11-133, 2012.

[9] W. An, and F. Shao, "The coverage-control optimization on sensor network subject to sensing area," Computer and Mathematics with Applications, vol. 57, no. 4, pp. 529-539, 2009.

[10] D. Zorbas, D. Glynos, P. Kotzanikolaou, and A. Komsia, "Solving coverage problems in wireless sensor networks using cover sets," Ad Hoc Networks, vol. 8, no. 4, pp. 400-415, 2010.

[11] J. Li, and H. Kao, "Distributed k-coverage self-location estimation scheme based on Voronoi diagram," IET Communications, vol. 4, no. 2 , pp. 167-177, 2010.

[12] M. Cardei, and D.Z. Du, "Improving wireless sensor network lifetime through power aware organization," Wireless Networks, vol. 6, no. 3, pp. 321-333, 2005.

[13] F. Zhao, J. Shin, and J. Reich. "Information driven dynamic sensor collaboration for tracking application," IEEE Signal Processing Magazine, vol. 61, no. 3, pp. 61-71, 2002.

[14] M. Mechitov, S. Sundresh, and Y. Kwon, "Cooperative tracking with binary detection sensor network," In: Proceeding of the $\mathrm{I} \mathrm{It}^{s t} \mathrm{In}$ ternational Conference on Embedded Networked Sensor Systems. vol. 4, no.1, pp. 332-341, 2003.

[15] S. Pattem, S. Poduri, and B. Krishnamachari, "Energy quality tradeoffs for target tracking in wireless sensor network," In: Proceeding of the $2^{\text {nd }}$ Workshop on Information Processing in Sensor Networks, vol. 15, no. 1, pp. 32-42, 2003.

[16] D. Tian, and N. D. Georgansa, "Anode scheduling scheme for energy conservation in large wireless sensor network," Wireless Communications and Mobile Computing, vol. 12, no. 3, pp. 271284, 2003.

[17] S. Zeyu, W. Weiguo, and W.A. Huanzhao, "Novel coverage algorithm based on event-probability-driven mechanism in wireless sensor network," Journal on Wireless Communications and Networking, vol.13, no. 4, pp. 1-12, 2014.

[18] K.F. Su, W.H. Ousiai, and H.C. Jiau, "Localization with mobile anchor points in wireless sensor networks," IEEE Transaction on Vehicular Technology, vol. 10, no. 3, pp. 1187-1196, 2005.

[19] H. Liu, P. Wan, C.W. Yi, X.H. Jia, and P. Niki, "Maximal lifetime scheduling in sensor surveillance networks," IEEE Transactions on Computers, vol. 11, no. 11, pp. 557-571, 2011. 